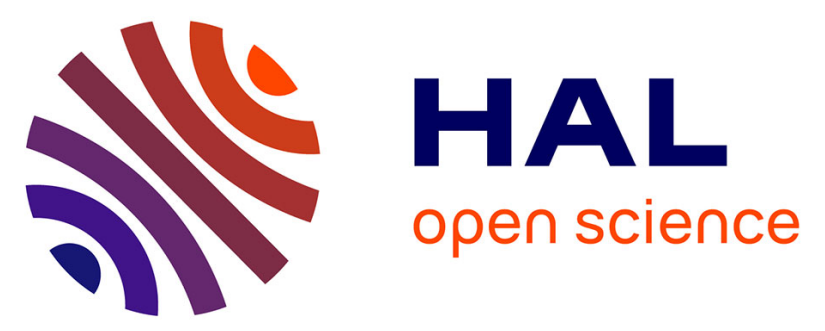

\title{
The Risk of Tumour Recurrence in Patients Undergoing Renal Transplantation for End-stage Renal Disease after Previous Treatment for a Urological Cancer: A Systematic Review
}

Romain Boissier, Vital Hevia, Harman Max Bruins, Klemens Budde, Arnaldo Figueiredo, Enrique Lledó-García, Jonathon Olsburgh, Heinz Regele, Claire Fraser Taylor, Rhana Hassan Zakri, et al.

\section{- To cite this version:}

Romain Boissier, Vital Hevia, Harman Max Bruins, Klemens Budde, Arnaldo Figueiredo, et al.. The Risk of Tumour Recurrence in Patients Undergoing Renal Transplantation for End-stage Renal Disease after Previous Treatment for a Urological Cancer: A Systematic Review. European Urology, 2018, pp.94 - 108. 10.1016/j.eururo.2017.07.017 . hal-01792732

\section{HAL Id: hal-01792732 \\ https://hal-amu.archives-ouvertes.fr/hal-01792732}

Submitted on 18 May 2018

HAL is a multi-disciplinary open access archive for the deposit and dissemination of scientific research documents, whether they are published or not. The documents may come from teaching and research institutions in France or abroad, or from public or private research centers.
L'archive ouverte pluridisciplinaire HAL, est destinée au dépôt et à la diffusion de documents scientifiques de niveau recherche, publiés ou non, émanant des établissements d'enseignement et de recherche français ou étrangers, des laboratoires publics ou privés. 
The risk of tumour recurrence in patients undergoing renal transplantation for endstage renal disease after previous treatment for a urological cancer: a systematic review

Romain Boissier ${ }^{1^{*}}$, Vital Hevia ${ }^{2 *}$, Harman Max Bruins ${ }^{3}$, Klemens Budde ${ }^{4}$, Arnaldo Figueiredo $^{5}$, Enrique Lledó García ${ }^{6}$, Jonathon Olsburgh ${ }^{7}$, Heinz Regele ${ }^{8}$, Claire Fraser Taylor $^{9}$, Rhana Hassan Zakri ${ }^{7}$, Cathy Yuhong Yuan ${ }^{10}$ and Alberto Breda ${ }^{11}$

\section{* These authors contributed equally and share the first authorship}

1. Aix-Marseille University, Marseille, France; Department of Urology \& Renal Transplantation, La Conception University Hospital, Assistance-Publique Marseille, France

2. Urology Department, Hospital Universitario Ramón y Cajal, Alcalá University, Madrid, Spain

3. Department of Urology, Radboudumc, Nijmegen, The Netherlands

4. Department of Nephrology, Charité Universitätsmedizin Berlin, Berlin, Germany

5. Department of Urology and Renal Transplantation, Coimbra University Hospital, Coimbra, Portugal

6. Department of Urology, Hospital General Universitario Gregorio Marañón, Madrid, Spain

7. Department of Urology \& Renal Transplantation Guy's and St Thomas' Hospital, London, UK

8. Clinical Institute of Pathology, Medical University of Vienna, Vienna, Austria

9. Department of Urology and renal Transplantation St George's Hospital, London, UK

10. Department of Medicine, Health Science Centre, McMaster University, Hamilton, Ontario, Canada 
11. Department of Urology, Fundacion Puigvert, University Autonoma of Barcelona, Barcelona, Spain 


\section{Corresponding author:}

Dr. Alberto Breda

Department of Urology and renal Transplantation

Puigvert Foundation

Carrer de Cartagena

08025 Barcelona

Spain

E-mail: albbred@gmail.com

Tel: 0034.934169700

Fax: 0034.934169730

Word count: Abstract 300 words, text 3750 words 


\section{Abstract (287 words)}

Context: Renal transplantation is the gold standard renal replacement therapy in end-stage renal disease (ESRD) owing to its superior survival and quality of life compared with dialysis. When the potential recipient has a history of cancer, the waiting period before RT is usually based on the Cincinnati Registry.

Objective: To systematically review all available evidence on the risk of cancer recurrence in ESRD patients with a history of urological cancer.

Evidence Acquisition: Medline, Embase and the Cochrane Library were searched up to March 2017 for all relevant publications reporting oncologic outcomes of urological cancer in patients who subsequently received a transplantation or remained on dialysis. The primary outcome was time to tumour recurrence. Secondary outcomes included cancer-specific and overall survival. Data were narratively synthesized in light of methodological and clinical heterogeneity. The risk of bias of each included study was assessed.

Evidence Synthesis: Thirty two retrospective studies enrolling 2,519 patients $(1,733$ dialyzed, 786 RT) were included. For renal cell carcinomas (RCC), the risks of recurrence, cancer-specific and overall survival were similar between transplantation and dialysis. For prostate cancer (PC), most of the tumours had favorable prognoses consistent with nomograms. Studies dealing with urothelial carcinomas (UC) mainly included upper urinary tract urothelial carcinoma (UUTUC) in the context of aristolochic acid nephropathy, for which the risks of synchronous bilateral tumour and recurrence were high. Data on testicular cancer were scarce. 
Conclusion: Immunosuppression after renal transplantation does not affect the outcomes and natural history of low risk RCC and PC. Therefore, the waiting time from successful treatment for these cancers to transplantation could be reduced. Except in the particular situation of aristolochic acid nephropathy, more studies are needed to standardize the waiting period after UC owing to the paucity of data.

Patient summary: Renal transplantation does not appear to increase the risk of recurrence of renal carcinoma or the recurrence of low-risk prostate cancer compared with dialysis. More reliable evidence is required to recommend a standard waiting period especially for urothelial and testicular carcinomas.

Keywords: Renal transplantation, Prostate cancer, Renal cancer, Urothelial carcinoma, Testicular cancer, Systematic review 


\section{MANUSCRIPT (3606 words)}

\section{Introduction}

Renal transplantation is the gold standard renal replacement therapy in end-stage renal disease (ESRD) owing to its superior survival and quality of life compared with other replacement therapies $(1,2)$.

The standard procedure for transplantation candidates includes systematic screening for the presence of any active/latent cancer or a history of cancer (3). In transplantation candidates with a previous history of urological cancer, it can be challenging to decide if patients are suitable for transplantation and if so how long the waiting period prior to transplantation should be. So far, the clinical decision has been mainly based on the Cincinnati Registry, which essentially considers the type of tumour and the time between its treatment and kidney transplantation (4). The waiting period varies from less than 2 years to at least 5 years according to the Registry. However, the Cincinnati Registry has several deficiencies: the treatment and the staging of the disease are not defined; the epidemiology of tumours, the diagnostic and therapeutic procedures/tests have changed and the prognostic tools have improved.

The objective of this systematic review was to appraise all available evidence on the risk of cancer recurrence in ESRD patients who underwent transplantation after having been successfully treated for a urological cancer. The primary objectives were to determine in patients with chronic kidney disease (CKD) 4/5 and a history of urological cancer, the risk of tumour recurrence after transplantation compared with renal replacement therapy (peritoneal and hemodialysis). The secondary objectives were to report on the overall and cancer-specific survival of transplanted and non-transplanted patients with a history of malignancy and to 
determine for each urological cancer the minimum tumour-free waiting period prior to transplantation.

\section{Evidence acquisition}

\subsection{Data sources and searches}

The systematic review was performed according to the Preferred Reporting Items for Systematic Reviews and Meta-Analyses (PRISMA) (5). The systematic review protocol was registered with

PROSPERO (http://www.crd.york.ac.uk/PROSPERO/display_record.asp?ID=CRD42016046867). Studies (January 1, 1995, to March 1, 2016) were identified by highly sensitive searches of electronic databases (Medline, Embase, Cochrane library databases). The initial literature search was performed in February 2016 and an updated search performed in March 2017. The search was complimented by additional sources including the reference lists of included studies. The search strategy is described in detail in Appendix 1.

\subsection{Study selection}

There was no restriction on types of study design. All randomized control studies (RCTs), non-randomized comparative studies (NRCS) and non-comparative studies (single-arm cohort studies, case reports) and meta-analyses published in the English language were included.

\subsection{Types of participants}

The study population was adults ( $\geq 18$ years) with CKD $4 / 5$ and previous urological cancer under renal replacement therapy or who subsequently undergo renal transplantation. 


\subsection{Data collection and data extraction}

Following de-duplication of abstracts, two reviewers (R.B. and V.H.) screened all abstracts and full-text articles independently. Disagreement was resolved by a third party (M.B.). References cited in all full-text articles were also assessed for additional relevant articles. A standardized data-extraction form was developed a priori to collect information on study design, patient characteristics (gender and age, type of urological cancer, baseline risk of tumour recurrence [based on stage, grade, histology or risk stratification using nomograms or risk groups]), interventions (duration of dialysis before cancer treatment, type and duration of immunosuppressive regimens, duration of tumour-free period prior to transplantation) and outcome measures (cancer recurrence, cancer-specific and overall survival).

\subsection{Risk of bias assessment}

Two reviewers (R.B. and V.H.) independently assessed the "risk of bias (RoB)" of each included study any discrepancies were revolved by a third reviewer (M.B.). The Cochrane Risk of Bias (RoB) tool was used for RoB assessment. For non-randomized studies the tool was modified to include an additional domain to assess the risk of confounding bias. A list of five important potential confounders was developed a priori with clinical content experts (European Association of Urology Renal Transplantation Guidelines Panel) (6,7). The confounders included were: type of urological cancer, baseline risk of tumour recurrence, means of diagnosis of tumour recurrence, duration of free period prior to transplantation and tumour-free status prior to transplantation. For each study, it was assessed whether each confounder was considered and whether, if necessary, the confounder was controlled in analysis. The RoB was considered to be high if the confounder had not been considered, was imbalanced between patients or not corrected for during analysis. This approach is detailed in the study protocol (ref PROSPERO). 


\subsection{Data synthesis}

Methodological and clinical heterogeneity of the included studies meant that meta-analysis was inappropriate, therefore, a narrative synthesis of the data was performed (https://www.york.ac.uk/crd/guidance/). The primary outcome was cancer recurrence at <1-year, 1-5-year and >5-year time points and is summarized in descriptive tables. Secondary outcomes were cancer-specific and the overall survival at < 1-year, 1-5-year and >5-year time points. Possible reasons for heterogeneity were explored using the available information such as differences in the population studied, the treatment given, or the way in which the outcomes were assessed. Intended formal subgroup analysis was not possible due to the inclusion of NRCS. Therefore, any subgroup differences were discussed narratively to explore potential effect size differences. A planned sensitivity analysis to assess the robustness of our review results, by repeating the analysis only including studies with an overall medium to low risk of bias, was not possible.

\section{Evidence synthesis}

\subsection{Quality of the studies}

A total of 1,891 studies were identified by the literature search and two reviewers screened all study abstracts independently. Of these, 53 studies were selected for full-text screening and 20 studies were eligible for inclusion. After an additional search in the references of these 20 studies and the elimination of duplicates with the selection of the most up-to-date publication for each series, 32 studies (4 retrospective comparative studies, 21 retrospective noncomparative studies and 7 case reports) were eligible for inclusion (Figure 1). The quality of studies was assessed as described above. Risk of bias is summarized in Figure 2. Overall there was a low risk of bias across all studies. Study design was retrospective for all studies. 


\subsection{Characteristics of studies, population and interventions (Table 1)}

In total 2,519 patients were included, suffering from: renal cell carcinoma (RCC) $(n=1,810$, $72 \%)$, prostate cancer $(\mathrm{PC})(\mathrm{n}=213,8 \%)$, upper urinary tract carcinoma/bladder cancer (UUTUC/BC) $(\mathrm{n}=451,18 \%)$ and testicular cancer $(\mathrm{TC})(\mathrm{n}=45,2 \%)$. The time on dialysis before cancer treatment ranged from 1 month to 14 years. Among these patients with a history of urological cancer, 1,733 (69\%) remained on dialysis, while 786 (31\%) had a transplantation with a waiting period ranging from 0 to $>5$ years.

\subsection{Oncological outcomes}

\subsubsection{Renal Cell Carcinoma}

Seventeen included studies evaluated patients suffering from RCC (Table 2). Mean age at diagnosis ranged from 37 to 57 years. The primary treatment was radical nephrectomy. Mean follow-up after nephrectomy was 3.6 years [ranging from 1.2-10.0 years]. The mean waiting period before transplantation ranged from 0 to 10 years. The recurrence rates for transplanted vs. dialyzed patients at $<1$ year, $1-5$ years and $>5$ years were $0-8 \%$ vs. $0 \%, 0-27 \%$ vs. $0-9 \%$ and $0-41 \%$ vs. $0-48 \%$ respectively. The 5-year cancer specific survival rates for transplantation vs. dialyzed patients were $79-100 \%$ vs. $77-100 \%$, respectively. Overall 5-year survival rates for transplantation vs. dialyzed patients were $80-100 \%$ vs. $76-100 \%$, respectively.

\subsubsection{Prostate cancer}

Eight included studies evaluated patients suffering from PC (Table 3). Mean age at diagnosis ranged from 57 to 69 years. Treatments were prostatectomy alone (61\%), prostatectomy + adjuvant radiotherapy (13\%), External Beam Radiotherapy (EBRT) (10\%), brachytherapy (1\%) and androgen deprivation therapy (7\%). The type of treatment was not specified in $8 \%$. 
Average follow-up after treatment ranged from 0.5 to 4 years. The mean interval between cancer treatment and transplantation ranged from 3 months to 4 years. Recurrence rates for transplantation patients at $<1$ year and $>5$ years were $0-9 \%$, and $4-20 \%$, respectively. The $1-$ 5-year cancer specific survival rates for transplantation patients ranged from $96 \%$ to $100 \%$. Overall 1-5-year survival rates for transplantation patients ranged from $62 \%$ to $100 \%$. No study reported results with a median follow-up beyond 5 years.

\subsubsection{Urothelial Carcinoma : Bladder cancer and Upper Urinary Tract Urothelial Carcinoma}

Nine studies evaluated patients suffering from UC (Table 4). Mean age on diagnosis ranged from 37 to 66 years. The treatments performed for UUTUC ranged from unilateral or bilateral nephroureterectomy to complete exenteration of the urinary tract. Bladder tumours were treated with endoscopic resection or cystectomy. Mean follow-up after treatment was 1.6 to 9 years. The interval between cancer treatment and transplantation was specified in one case report: 6 years.

Five-year recurrence rates for transplantation and dialyzed patients were $4.2-29 \%$ vs. $24-57 \%$, respectively. Five-year cancer specific survival rates and overall survival were $86-98 \%$ and $43-88 \%$, respectively for patients who remained on dialysis. Cancer specific and overall survival rates were not reported for transplanted patients.

\subsubsection{Testicular cancer (Table 5)}

Three studies evaluated patients suffering from TC (Table 5)(4,8). The 5-year recurrence rate was $5 \%$. The waiting period before transplantation was not defined. Histology and grade were specified only in 2 case reports: stage I seminoma and teratoma. Cancer-specific and overall survivals of $100 \%$ at $1-5$ years were reported in the 2 case reports. 


\subsection{Risk of bias and confounding}

The risk of bias and confounding was relevant, in particular in the light of the generally low level of evidence studies (Figure 2). The type of urological malignancy and the risk of recurrence were taken into account in most of the studies. The duration of the tumour-free period to transplantation and the confirmation of a tumour-free status prior to transplantation were relevant only for the studies with an intervention arm (transplantation).

\section{Discussion}

\subsection{Principal findings}

For RCC, the risk of recurrence was similar between transplantation and dialysis. Stage, grade, histological subtype and solid/cystic component of the tumour were the main prognostic factors for recurrence.

For PC, data were too scarce to reach a conclusion on the impact of transplantation on the risk of recurrence because the majority of the included studies were non-comparative and involved only transplanted patients. Except in the study of Woodle et al which is the last update of the Cincinnati Registry, studies included mainly PC with favorable prognosis: low stage, low grade and low recurrence rates consistent with nomograms (D'Amico, Partin, Kattan, MSKCC) (9).

For UC, the studies mainly included UUTUC in the context of aristolochic acid nephropathy. In this specific situation, the rate of synchronous bilateral tumour was $10 \%$ to $16 \%$ and the rate of contralateral recurrence was $31 \%$ to $39 \%$. Data on bladder UC and TC were scarce. For TC, one case report highlighted the possibility of late recurrence (2.3 years) even for a stage I seminoma. 


\subsection{Findings in the context of existing evidence}

Organ transplantation is a risk factor of cancer recurrence especially for viral-induced cancers. However, this risk is modulated by the type of cancer and the type of transplant (10). In a large cohort of solid organ transplant recipients (1970-2008), Brattströmt et al. reported a $30 \%$ increased mortality risk for recipients with a history of cancer. However, this risk was moderately increased for renal transplant recipients (Hazard Ratio [HR] 1.2\% 95\%: 1.0-1.4, $p$ $<0.05)$ and mainly concerned recipients of other organs (HR 1.8) (11).

An active cancer is a contraindication for transplantation and a patient with (except active surveillance for prostate cancer) a history of malignancy must be in remission before transplantation. Pre-transplant evaluation includes a systematic search for sub-clinical active or latent tumours. For patients with a history of treated malignancy, the waiting period before transplantation varies from 2 to 5 years after cancer treatment. These recommendations are based on the Israel Penn International Transplant Tumour Registry $(4,9)$.

However, these studies from ten years ago now have several shortcomings and may no longer reflect the epidemiology of patients currently seen in pre- transplantation evaluation. Several oncological data were missing: stage, histological subtype, grade, and type of treatment. For PC, the Penn et al. study essentially included T3 tumours diagnosed in the pre-prostatespecific antigen era. For RCC, the tumours were mainly large and symptomatic. For bladder tumours, the stage, grade, multifocality, type of adjuvant intravesical instillation and the distinction between non-muscle invasive and muscle invasive tumours were not reported (4).

\subsubsection{RCC and CKD}

In this systematic review, the main histological sub-type was clear cell RCC (53-79\%), followed by tubulo-papillary RCC (17-50\%). The tumours were mainly of low stage (78$100 \%$ of pT1), and/or low grade (51-92\% grade I-II) (Table 2). 
RCC diagnosed in a context of CKD may have a better prognosis for several reasons. ESRD patients are often monitored more closely than the normal population, favoring early diagnosis with smaller size and lower grade. The main risk factor of RCC is Acquired Cystic Kidney Disease (ACKD), which increases with the duration of dialysis (12). RCC is more common for patients with CKD compared with the general population. The standardized incidence ratio of RCC in dialysis patients is 14 to 17 times higher than that in the general population (13). The systematic review data were consistent with the current knowledge on RCC in CKD patients, reporting an increased prevalence of low-grade tumours (51\%-100\%) and the papillary subtype (17\%-37\%) Table 3) (14-16). The duration of dialysis alters the histological spectrum of tumours: clear cell RCC is the predominant subtype for patients with short dialysis duration, papillary RCC being the predominant type in patients on dialysis for more than 4 years (14).

\subsubsection{Prostate cancer and CKD}

In this systematic review, the series included were mainly cohorts of patients who were transplanted after their PC treatment (111 transplanted patients vs. 6 patients who remained on dialysis) with mainly localized PC of excellent prognosis (D'Amico low to intermediate risk or stage $\leq \mathrm{II})$. By contrast, in 2005 the report of Woodle et al. included $12 \%$ with stage III PC. In 2001 Chapman et al. did not report the histology and prognosis of the patients included (9) (Table 3).

To date, there is no evidence of worse prognosis for PC in ESRDs. Although the increased prevalence of hypogonadism was suspected to induce PC of worse prognosis, there is no study validating this hypothesis (17). It was not demonstrated that the prognostic performance of pre-therapy (D'Amico, Partin) and post-therapy (Kahn, Kattan) nomograms is altered for CKD patients $(18,19)$. 


\subsubsection{Urothelial carcinoma and CKD}

In this systematic review, the most common tumour site of UC was the upper urinary tract (57\% to $100 \%$ of the case series). The prevalence of a synchronous bladder tumour was $42-$ $81 \%$ and the prevalence of a bilateral tumour was $10-16 \%(20,21)$. Concerning the stage, $72 \%$ [56\%-100\%] of the patients were diagnosed with a non-muscle invasive tumour. All publications followed the three-stage classification according to the 1973 World Health Organization classification with a high-grade distribution ranging from $15 \%$ to $69 \%$ (Table 4).

There were few studies on bladder cancer. Among them, tumour stage (tumour invading muscle or not) and use of adjuvant intravesical therapy were rarely mentioned.

Most studies dealing with UUTUC concerned patients with aristolochic nephropathy and seemed to have a higher risk of recurrence and multifocality than UUTUC induced by tobacco and toxic substances. (22)

\subsubsection{Testicular cancer}

We found no comparative study concerning TC. Testicular cancer had a low risk of recurrence according to Penn et al (4). A single clinical case indicated the possibility of recurrence for stage I seminoma (8).

\subsection{Implications for practice}

Considering the rates of recurrence and the prognostic factors of recurrence reported in this systematic review of the literature, it seems possible to optimize the waiting period between treatment for cancer and transplantation. 


\subsubsection{Renal Cell Carcinoma}

The main prognostic factors of recurrence identified in this systematic review were: stage, tumour size, Fuhrman grade, tumour solid nature, symptomatic tumours on diagnosis, Conventional renal cell carcinoma (CRCC) subtype and lymph node involvement (Table 2).

This systematic review reported a significant risk of recurrence even for low-stage and lowgrade tumours. In Sheashaa et al. and Denton et al, in particular, where ipsilateral nephrectomy was performed systematically during transplantation, there was a risk of recurrence of up to $25 \%$ at 5 years, even though these were low-grade and low-stage infraradiological tumours $(23,24)$. In these studies, contralateral involvement occurred in up to $36 \%$ of the cases. Recurrences were essentially recurrent/second contralateral RCC with an early diagnosis. Cancer-specific and overall survival rates were similar between dialyzed and transplanted patients. (Table 2).

The conclusion of this systematic review highlights the requirement for regular annual monitoring of the native kidney in CKD patients and particularly those with a history of RCC. The reference treatment for a cancer of the native kidneys is radical nephrectomy.

\subsubsection{Prostate cancer}

In this systematic review, the main prognostic factors for recurrence were: stage, PSA and Gleason score. Radical prostatectomy is the preferred treatment both for staging and curative purposes. Lymph node resection may be best limited to one side of the pelvis to preserve the iliac vessels for the transplantation on the other side (25). Transperineal prostatectomy could also be an option to preserve the iliac vessels (26).

The studies reported low recurrence rates consistent with prognostic nomograms. In the Tillou et al. study, the risk of biochemical recurrence at 10 years, calculated using the MSKCC 
nomogram did not exceed $3 \%$ in the worst case and was $1 \%-2 \%$ in all other case. This nomogram has proven its reliability in other studies on large cohorts (27). The nomogram allowed registration of patients on the transplantation waiting list at an earlier stage whilst radical prostatectomy made the decision to put the patient on the transplant list easier. The authors reported no recurrence with a mean follow-up of 3.2 years, suggesting that there could be no waiting period for transplantation in cases of cured low-risk PC (25).

In their study, Woodle et al. (which was an update of the PC series published by Penn et al. in 1995 and 1997) included a significant number of high-risk extraprostatic tumours. Despite its shortcomings, this study is still unequaled for the study of high-risk prostate cancer before transplantation (11).

\subsubsection{Urothelial carcinoma (Bladder and UUTUC)}

The included studies showed that UUTUC has a high-risk of recurrence. The risk of synchronous bilateral involvement is $10 \%-16 \%$ and the risk of contralateral recurrence is 31\%-39\% (Table 4). For candidates for transplantation with a history of UUTUC, two strategies are justified:

1. Systematic treatment of the contralateral upper urinary tract and/or the bladder by nephroureterectomy and/or even cystectomy;

2. Close monitoring of the bladder and the contralateral upper urinary tract.

\subsubsection{Testicular cancer}

The level of evidence reported in this study did not allow us to conclude on the risk of testicular tumour recurrence after renal transplantation. The 2 case-reports highlighted the risk 
of retroperitoneal recurrence after transplantation, even with a long waiting period (Table 5). A case-by-case discussion is needed to decide on a period of exclusion before transplantation.

\subsection{Implications for research}

Although the findings of this systematic review enabled us to identify the prognostic factors of recurrence and to conclude that immunosuppression did not modify the natural history of urological cancer for selected patients, the literature in this particular area differed according to the type of cancer. With the exception of Tillou et al. (19 patients), we found no comparative study for $\mathrm{PC}(25)$. There were few studies on bladder cancer, with no data concerning the stage (tumour invading the vesical muscle or not) and the use of adjuvant intravesical therapy.

In practice, it appears to be difficult to evaluate the excess risk of recurrence represented by the initiation of immunosuppressive therapy and thus the possible deleterious impact of transplantation on recurrence and cancer-specific survival. Randomized controlled trials will ethically and logistically be difficult to conduct. However, well-designed prospective cohort studies with homogenous type/stage of cancer and clear predefined oncological outcomes at different time points are needed to strongly support a reduction of the waiting period for low risk RCC and PC, which was a tendency suggested in this systematic review.

\subsection{Limitations of this study}

This report is the first systematic review assessing and appraising all available evidence of the risk of cancer recurrence for CKD patients on dialysis or who have undergone transplantation. Limitations mainly consist in the low level of the references: 
- The included studies are all retrospective and most of them are not comparative. As such, the risk of bias and/or confounding is high in most studies.

- Long-term follow-up is lacking. Several data were not systematically reported: prognostic score or monograms, duration and type of immunosuppressive treatment, type of recurrence (local or systemic).For PC, the oncological results of nontransplanted patients were based on only 6 patients in 2 studies.

- For urothelial tumours, the majority of studies included UUTUC occurring in the particular field of aristolochic acid nephropathy.

\section{Conclusions}

Although this systematic review summarized all the available evidence on renal transplantation and history of cancer, it was limited by the level of evidence of the included studies, which mainly consisted of non-comparative retrospective cohort studies of preselected patients. Acknowledging that the comparison is not free from bias, this systematic review indicates that immunosuppression does not seem to alter the natural history of recurrence and mortality for low-risk renal and prostate cancer, and could lead to a shortening of the waiting period in this specific situation. For high-risk renal and prostate cancer, the historical Cincinnati registry is still an unequalled source of data. Although this registry study suffers from many shortcomings, waiting period should not be modified for high-risk renal and prostate cancers. Urothelial carcinomas are multifocal and highly recurrent tumours. For transplantation candidates, close follow-up or systematic bilateral nephroureterectomy are both possible strategies. For patients with a history of TC a case-by-case discussion is recommended. 
Acknowledgments: The valuable help of European Association of Urology Guidelines Office, Arnhem, Netherlands in providing the full text articles and administrative support is gratefully acknowledged. Furthermore, we thank Thomas Lamand Cathy Yuhong Yuan of the EAU Guidelines Office Methods Committee, for providing methodological support

\section{Conflict of interest disclosures:}

None related to the present work

\section{Funding/Support:}

This study was supported by the European Association of Urology.

Role of the sponsor: The European Association of Urology had no role in the design and conduct of the study, collection, management, analysis, and interpretation of the data, preparation, review, or approval of the manuscript nor the decision to submit the manuscript for publication. 
Appendix 1: Full search strategy.

1 exp kidney transplantation/

2 ((renal or kidney) adj3 (transplant* or allotransplant* or grafting homotransplant* or retransplant*)).tw,kw.

31 or 2

4 ((urological or urinary tract or prostate or prostatic or urothel* or ureter* or transitional cell or kidney* or renal or bladder or penis or penile or testicular or testis or testes) adj3 (cancer* or carcin* or malig* or tumour* or tumour* or neoplas*)).tw,kw.

5 exp transitional cell carcinoma/ or exp Carcinoma, Transitional Cell/

6 exp Ureteral Neoplasms/ or exp ureter tumour/

7 exp Kidney Neoplasms/ or exp kidney tumour/

8 exp bladder tumour/ or exp Urinary Bladder Neoplasms/

9 exp Prostatic Neoplasms/ or exp prostate tumour/

10 exp penis tumour/ or exp Penile Neoplasms/

11 exp testis tumour/ or exp Testicular Neoplasms/

12 or/4-11

$13 \quad 3$ and 12

14 exp chronic kidney disease/ or exp Renal Insufficiency, Chronic/

15 (chronic and (kidney or renal) and (end stage* or (stage* and ("4/5" or "4" or "5" or "4$5 ")))) . \mathrm{tw}, \mathrm{kw}$.

16 (Chronic and (kidney or renal) and (disease* or disorder* or dysfunction* or insufficien* or failure*)).tw,kw.

17 exp renal replacement therapy/ or exp Renal Dialysis/

18 ((Renal or kidney) adj3 (dialysis or dialyses or replacement or support)).tw,kw.

19 (hemodialys?s or extracorporeal dialys?s).tw,kw.

20 or/14-19

$21 \quad 13$ and 20

22 ((exp animals/ or exp animal/ or exp nonhuman/ or exp animal experiment/ or animal model/ or animal tissue/ or non human/) not (humans/ or human/)) or ((rats or mice or mouse or cats or dogs or animal* or cell lines) not (human* or men or women)).ti.

$23 \quad 21$ not 22

24 ((child/ or Pediatrics/ or Adolescent/ or Infant/ or adolescence/ or newborn/) not (adult/ or aged/)) or ((baby or babies or child or children or pediatric* or paediatric* or peadiatric* or infant* or infancy or neonat* or newborn* or new born* or kid or kids or adolescen* or preschool or pre-school or toddler*) not (aged or adult* or elder* or senior or men or women)).ti. $25 \quad 23$ not 24

26 limit 25 to $\mathrm{yr}=" 1995$-Current"

27 remove duplicates from 26 


\section{References}

1. Laupacis A, Keown P, Pus N, Krueger H, Ferguson B, Wong C, et al. A study of the quality of life and cost-utility of renal transplantation. Kidney Int. 1996 Jul;50(1):235-42.

2. Wolfe RA, Ashby VB, Milford EL, Ojo AO, Ettenger RE, Agodoa LY, et al. Comparison of mortality in all patients on dialysis, patients on dialysis awaiting transplantation, and recipients of a first cadaveric transplant. N Engl J Med. 1999 Dec 2;341(23):1725-30.

3. Kälble T, Lucan M, Nicita G, Sells R, Burgos Revilla FJ, Wiesel M, et al. EAU guidelines on renal transplantation. Eur Urol. 2005 Feb;47(2):156-66.

4. Penn I. Evaluation of transplant candidates with pre-existing malignancies. Ann Transplant. 1997;2(4):14-7.

5. Moher D, Liberati A, Tetzlaff J, Altman DG, PRISMA Group. Preferred reporting items for systematic reviews and meta-analyses: the PRISMA statement. PLoS Med. 2009 Jul 21;6(7):e1000097.

6. Higgins JPT. Green S. Cochrane handbook for systematic reviews of interventions version 5.1. 0. Cochrane Collab. 2011;5(0).

7. Reeves BC, Deeks JJ, Higgins JP. 13 Including non-randomized studies. Cochrane Handb Syst Rev Interv. 2008;1:391.

8. Dean C, Bloomfield D, Holt S. The challenge of germ cell tumour therapy in dialysis and transplantation. Nephrol Dial Transplant Off Publ Eur Dial Transpl Assoc - Eur Ren Assoc. 2005 Dec;20(12):2867-8.

9. Woodle ES, Gupta M, Buell JF, Neff GW, Gross TG, First MR, et al. Prostate cancer prior to solid organ transplantation: the Israel Penn International Transplant Tumour Registry experience. Transplant Proc. 2005 Mar;37(2):958-9.

10. Viecelli AK, Lim WH, Macaskill P, Chapman JR, Craig JC, Clayton P, et al. CancerSpecific and All-Cause Mortality in Kidney Transplant Recipients With and Without Previous Cancer. Transplantation. 2015 Dec;99(12):2586-92.

11. Brattström C, Granath F, Edgren G, Smedby KE, Wilczek HE. Overall and cause-specific mortality in transplant recipients with a pretransplantation cancer history. Transplantation. 2013 Aug 15;96(3):297-305.

12. Farivar-Mohseni H, Perlmutter AE, Wilson S, Shingleton WB, Bigler SA, Fowler JE. Renal cell carcinoma and end stage renal disease. J Urol. 2006 Jun;175(6):2018-20; discussion 2021.

13. Ishikawa I. Acquired cystic disease of the kidney and renal cell carcinoma-complication of long-term hemodialysis. In: Acquired cystic disease of the kidney and renal cell carcinomacomplication of long-term hemodialysis. Springer. 2007. 
14. Breda A, Lucarelli G, Luccarelli G, Rodriguez-Faba O, Guirado L, Facundo C, et al. Clinical and pathological outcomes of renal cell carcinoma (RCC) in native kidneys of patients with end-stage renal disease: a long-term comparative retrospective study with RCC diagnosed in the general population. World J Urol. 2015 Jan;33(1):1-7.

15. Neuzillet Y, Tillou X, Mathieu R, Long J-A, Gigante M, Paparel P, et al. Renal cell carcinoma (RCC) in patients with end-stage renal disease exhibits many favourable clinical, pathologic, and outcome features compared with RCC in the general population. Eur Urol. 2011 Aug;60(2):366-73.

16. Gigante M, Neuzillet Y, Patard J-J, Tillou X, Thuret R, Branchereau J, et al. Renal cell carcinoma (RCC) arising in native kidneys of dialyzed and transplant patients: are they different entities? BJU Int. 2012 Dec;110(11 Pt B):E570-3.

17. Butler AM, Olshan AF, Kshirsagar AV, Edwards JK, Nielsen ME, Wheeler SB, et al. Cancer incidence among US Medicare ESRD patients receiving hemodialysis, 1996-2009. Am J Kidney Dis Off J Natl Kidney Found. 2015 May;65(5):763-72.

18. Mottet N, Bellmunt J, Bolla M, Briers E, Cumberbatch MG, De Santis M, et al. EAUESTRO-SIOG Guidelines on Prostate Cancer. Part 1: Screening, Diagnosis, and Local Treatment with Curative Intent. Eur Urol. 2016 Aug 25;

19. Breyer BN, Whitson JM, Freise CE, Meng MV. Prostate cancer screening and treatment in the transplant population: current status and recommendations. J Urol. 2009 May;181(5):2018-25; discussion 2025-6.

20. Wu C-F, Chang P-L, Chen C-S, Chuang C-K, Weng H-H, Pang S-T. The outcome of patients on dialysis with upper urinary tract transitional cell carcinoma. J Urol. 2006 Aug;176(2):477-81.

21. Kang C-H, Chen C-H, Chiang P-H. Primary urothelial carcinoma of the upper urinary tract in dialysis patients with 5-year follow-up. Jpn J Clin Oncol. 2010 Mar;40(3):241-6.

22. Rouprêt M, Babjuk M, Compérat E, Zigeuner R, Sylvester RJ, Burger M, et al. European Association of Urology Guidelines on Upper Urinary Tract Urothelial Cell Carcinoma: 2015 Update. Eur Urol. 2015 Nov;68(5):868-79.

23. Sheashaa HA, Rennke HG, Bakr MA, Abbas TM, Atta AF, Sangak A, et al. Impact of accidental discovery of renal cell carcinoma at time of renal transplantation on patient or graft survival. Transplantation. 2011 Nov 27;92(10):1123-8.

24. Denton MD, Magee CC, Ovuworie C, Mauiyyedi S, Pascual M, Colvin RB, et al. Prevalence of renal cell carcinoma in patients with ESRD pre-transplantation: a pathologic analysis. Kidney Int. 2002 Jun;61(6):2201-9.

25. Tillou X, Chahwan C, Le Gal S, Bensadoun H, Doerfler A. Prostatectomy for localized prostate cancer to prepare for renal transplantation in end-stage renal disease patients. Ann Transplant. 2014;19:569-75. 
26. Yiou R, Salomon L, Colombel M, Patard JJ, Chopin D, Abbou CC. Perineal approach to radical prostatectomy in kidney transplant recipients with localized prostate cancer. Urology. 1999 Apr;53(4):822-4.

27. Ryosaka M, Ishida H, Takagi T, Shimizu T, Tanabe K, Kondo T. Solid-type RCC originating from native kidneys in renal transplant recipients should be monitored cautiously. Transpl Int Off J Eur Soc Organ Transplant. 2015 Jul;28(7):813-9.

28. Savaj S, Liakopoulos V, Ghareeb S, Musso C, Sahu K, Bargman JM, et al. Renal cell carcinoma in peritoneal dialysis patients. Int Urol Nephrol. 2003;35(2):263-5.

29. Joshi R, Mammen K, Pawar B. Successful renal transplantation after two separate urinary tract malignancies. Indian J Urol IJU J Urol Soc India. 2008 Apr;24(2):256-7.

30. Takagi T, Kondo T, Okumi M, Ishida H, Tanabe K. Differences in Clinical and Pathological Features of Renal Cell Carcinoma Between Japanese Patients After Kidney Transplantation and Those on Hemodialysis. Ther Apher Dial Off Peer-Rev J Int Soc Apher Jpn Soc Apher Jpn Soc Dial Ther. 2017 Apr;21(2):133-8.

31. Shrewsberry AB, Osunkoya AO, Jiang K, Westby R, Canter D, Pattaras J, et al. Renal cell carcinoma in patients with end-stage renal disease has favorable overall prognosis. Clin Transplant. 2014 Feb;28(2):211-6.

32. Hora M, Hes O, Reischig T, Urge T, Klecka J, Ferda J, et al. Tumours in end-stage kidney. Transplant Proc. 2008 Dec;40(10):3354-8.

33. Kojima Y, Takahara S, Miyake O, Nonomura N, Morimoto A, Mori H. Renal cell carcinoma in dialysis patients: a single center experience. Int J Urol Off J Jpn Urol Assoc. 2006 Aug;13(8):1045-8.

34. Peces R, Martínez-Ara J, Miguel JL, Arrieta J, Costero O, Górriz JL, et al. Renal cell carcinoma co-existent with other renal disease: clinico-pathological features in pre-dialysis patients and those receiving dialysis or renal transplantation. Nephrol Dial Transplant Off Publ Eur Dial Transpl Assoc - Eur Ren Assoc. 2004 Nov;19(11):2789-96.

35. Stiles KP, Moffatt MJ, Agodoa LY, Swanson SJ, Abbott KC. Renal cell carcinoma as a cause of end-stage renal disease in the United States: patient characteristics and survival. Kidney Int. 2003 Jul;64(1):247-53.

36. Gulanikar AC, Daily PP, Kilambi NK, Hamrick-Turner JE, Butkus DE. Prospective pretransplant ultrasound screening in 206 patients for acquired renal cysts and renal cell carcinoma. Transplantation. 1998 Dec 27;66(12):1669-72.

37. Shingleton WB, Blalock J, Clark J, Bigler SA. Renal cell carcinoma in native kidneys of patients with end stage renal disease. J Miss State Med Assoc. 1998 Mar;39(3):86-9.

38. Chahwan C, Doerfler A, Brichart N, Bouyé S, Culty T, Iselin C, et al. Prostate cancer before renal transplantation: A multicentre study. Progres En Urol J Assoc Francaise Urol Soc Francaise Urol. 2017 Mar;27(3):166-75. 
39. Schonberger B. [Bladder dysfunction and surgery in the small pelvis. Therapeutic possibilities]. Urol Ausg A. 2003 Dec;42:1569-75.

40. Özçelik Ü, Bircan HY, Karakayalı F, Moray G, Demirağ A. Delaying Renal Transplant after Radical Prostatectomy for Low-Risk Prostate Cancer. Exp Clin Transplant Off J Middle East Soc Organ Transplant. 2015 Nov;13 Suppl 3:74-6.

41. Kocak B, Bilen CY, Dilek M, Adibelli Z, Akpolat T, Sarikaya S. Is localized prostate cancer an obstacle for an immediate consideration for renal transplantation? A case report. Transplant Proc. 2009 Jun;41(5):1961-2.

42. Chang $\mathrm{C}-\mathrm{H}$, Yang C-M, Yang A-H. Renal diagnosis of chronic hemodialysis patients with urinary tract transitional cell carcinoma in Taiwan. Cancer. 2007 Apr 15;109(8):1487-92.

43. Hung P-H, Shen C-H, Chiu Y-L, Jong I-C, Chiang P-C, Lin C-T, et al. The aggressiveness of urinary tract urothelial carcinoma increases with the severity of chronic kidney disease. BJU Int. 2009 Nov;104(10):1471-4.

44. Li C-E, Chien C-S, Chuang Y-C, Chang Y-I, Tang H-P, Kang C-H. Chronic kidney disease as an important risk factor for tumour recurrences, progression and overall survival in primary non-muscle-invasive bladder cancer. Int Urol Nephrol. 2016 Jun;48(6):993-9.

45. Ou JH, Pan CC, Lin JS, Tzai TS, Yang WH, Chang CC, et al. Transitional cell carcinoma in dialysis patients. Eur Urol. $2000 \mathrm{Jan} ; 37(1): 90-4$.

46. Chapman JR, Sheil AG, Disney AP. Recurrence of cancer after renal transplantation. Transplant Proc. 2001 Mar;33(1-2):1830-1.

47. Juric I, Basic-Jukic N. Testicular Seminoma Occurring After Kidney Transplantation in a Patient Previously Treated for Teratoma: De Novo Malignancy or Recurrence in a Different Histologic Form? Transplant Proc. 2016 Nov;48(9):3128-9. 